\title{
A NEW METHOD FOR NUMERICAL SOLUTION OF CHECKERBOARD FIELDS
}

\section{STEIN A. BERGGREN, DAG LUKKASSEN, ANNETTE MEIDELL, AND LEON SIMULA}

Received 6 April 2001 and in revised form 4 September 2001

We consider a generalized version of the standard checkerboard and discuss the difficulties of finding the corresponding field by standard numerical treatment. A new numerical method is presented which converges independently of the local conductivities.

\section{Introduction}

Very few microstructures yield explicit formulae for their effective conductivity. One type of such structures is checkerboards. By using a duality argument Dychne [6] proved about 30 years ago the famous formula for the effective conductivity

$$
\lambda_{\text {eff }}=\sqrt{\lambda_{\mathrm{g}} \lambda_{w}}
$$

for a standard checkerboard of conductivity $\lambda_{g}$ and $\lambda_{w}$. The explicit solution of the corresponding temperature-field was later found by Berdichevskii [1]. In particular he found that the heat-flux is infinitely high in the corners of the squares. Subsequently, explicit solutions for rectangular and triangular checkerboards were found in $[17,19,20]$.

Mortola and Steffé [18] presented in 1985 an interesting conjecture concerning the effective conductivity of four phase checkerboards. Many attempts were made to prove/disprove the conjecture, even by specialists in homogenization theory (see [22]), but the problem remained unsolved for the rest of the century. Very recently the conjecture has been proved by Craster and Obnosov [5] and independently by Milton [16] (using a completely different proof).

There are many interesting works on other types of checkerboards. Concerning three-dimensional checkerboards, we refer to [12, 15] (see also [14]). 
Random checkerboards where studied in [2, 13, 7, 21]. A new type has recently been considered in [10] where the conductivity blows up at the corners in a diagonal direction and degenerates in the orthogonal direction. There appears to be two natural ways of defining the effective conductivity for this problem in terms of variational problems involving weighted Sobolev spaces. However, the corresponding effective conductivities are very different (Lavrentiev phenomenon). Similar observations have been done for nonlinear checkerboards in [23], which also contains a generalization of the Dykhne formula to power-law materials.

Due to the behavior of the solutions near the corner points it is difficult to solve the corresponding variational problems by usual numerical methods, even for the standard checkerboard. In this paper, we consider a generalized version of the standard checkerboard and focus on these difficulties, both theoretically and experimentally (numerical experiments). Moreover, we present a new numerical method for determining the corresponding field which converges in the energy norm independent of the local conductivities.

\section{The model problem}

We consider the stationary heat conduction problem for the checkerboard structure in Figure 2.1, where the conductivity $\lambda(x)$ in each quarter $V$ of a period is given by

$$
\lambda(x)= \begin{cases}k_{g} l(r) & \text { on grey parts, } \\ k_{w}(l(r))^{-1} & \text { on white parts, }\end{cases}
$$

where $0<\beta \leq l(r) \leq \gamma<\infty$ for some constants $\beta$ and $\gamma, r$ is the distance from the center of $V$, and $l$ is continuous at $r=0$. Due to symmetries it is enough to only consider the set $\mathrm{V}$ for the calculation of the effective conductivity $\lambda_{\text {eff. }}$ This value can be determined by the following variational formula:

$$
\lambda_{\text {eff }}=\frac{1}{|V|} \min _{u \in W}\left\{\int_{V} \lambda(x)\left|\operatorname{grad} u+e_{1}\right|^{2} d x\right\}
$$

or equivalently by (cf. [9])

$$
\frac{1}{\lambda_{\text {eff }}}=\frac{1}{|V|} \min _{u \in W}\left\{\int_{V} \frac{1}{\lambda(x)}\left|\operatorname{grad} u+e_{1}\right|^{2} d x\right\},
$$

where

$$
W=\left\{u \in W^{1,2}(V): u\left(-1, x_{2}\right)=0, u\left(1, x_{2}\right)=0\right\},
$$

where $e_{1}=[1,0]$. By (2.2) and (2.3) we can obtain the formula

$$
\lambda_{\mathrm{eff}}=\sqrt{\mathrm{k}_{w} \mathrm{k}_{\mathrm{g}}}
$$



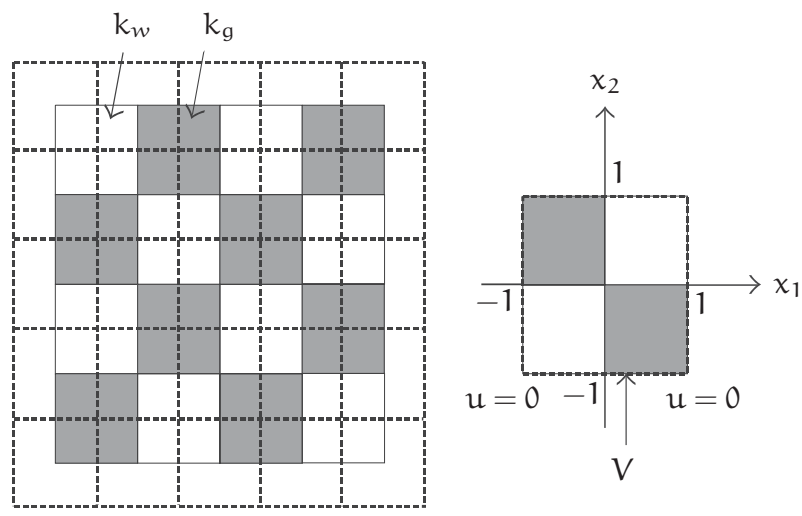

Figure 2.1. A part of a checkerboard with a quarter of a period, denoted $\mathrm{V}$, shown to the right.

which was first proved by Dychne [6]. If $W^{\prime}$ is any subspace of $W$ and $\lambda_{\text {eff }}^{+}$ and $\lambda_{\text {eff }}^{-}$are the values defined by

$$
\begin{aligned}
& \lambda_{\text {eff }}^{+}=\frac{1}{|V|} \min _{u \in W^{\prime}}\left\{\int_{V} \lambda(x)\left|\operatorname{grad} u+e_{1}\right|^{2} d x\right\}, \\
& \frac{1}{\lambda_{\text {eff }}^{-}}=\frac{1}{|V|} \min _{u \in W^{\prime}}\left\{\int_{V} \frac{1}{\lambda(x)}\left|\operatorname{grad} u+e_{1}\right|^{2} d x\right\},
\end{aligned}
$$

then it is clear that $\lambda_{\text {eff }}^{-} \leq \lambda_{\text {eff }} \leq \lambda_{\text {eff }}^{+}$.

\section{Solutions found in finite-dimensional spaces}

The minimizer $u^{+}$of (2.6) is an approximation of the minimizer $u$ of (2.2). When the ratio $k_{g} / k_{w}$ is large, it becomes almost impossible to obtain good approximations by using the finite element method (FEM). In fact we have the following result.

Theorem 3.1. If $W^{\prime}$ is a finite-dimensional subspace of $W$, then

$$
\frac{\lambda_{\text {eff }}^{+}}{\lambda_{\text {eff }}} \longrightarrow \infty \quad \text { as } \frac{k_{\mathrm{g}}}{k_{w}} \longrightarrow \infty .
$$

Remark 3.2. As a consequence we obtain that $\left\|\mathfrak{u}-\mathfrak{u}^{+}\right\|_{\lambda}^{2} / \lambda_{\text {eff }} \rightarrow \infty$ as $k_{\mathrm{g}} / k_{w} \rightarrow \infty$, where $\|v\|_{\lambda}$ denotes the energy norm

$$
\|v\|_{\lambda}=\sqrt{\frac{1}{|\mathrm{~V}|} \int_{V} \lambda(x)|\operatorname{grad} v|^{2} \mathrm{~d} x .}
$$


This follows since

$$
\begin{aligned}
\left\|u-u^{+}\right\|_{\lambda} & =\left\|u+x_{1}-\left(u^{+}+x_{1}\right)\right\|_{\lambda} \geq\left\|u^{+}+x_{1}\right\|_{\lambda}-\left\|u+x_{1}\right\|_{\lambda} \\
& =\sqrt{\lambda_{\text {eff }}^{+}}-\sqrt{\lambda_{\text {eff }}}=\sqrt{\lambda_{\text {eff }}}\left(\sqrt{\frac{\lambda_{\text {eff }}^{+}}{\lambda_{\text {eff }}}-1}\right) .
\end{aligned}
$$

Proof. Let $c=\min \{l(r)\}$ and let $\Omega_{\mathrm{g}}$ be the grey area of $\mathrm{V}$. Since

$$
c k_{g} \int_{\Omega_{g}}\left|\mathrm{D} u+e_{1}\right|^{2} \mathrm{~d} x \leq \int_{\Omega_{g}} \lambda(x)\left|\mathrm{D} u+e_{1}\right|^{2} \mathrm{~d} x \leq \int_{V} \lambda(x)\left|\mathrm{D} u+e_{1}\right|^{2} \mathrm{~d} x
$$

we obtain that

$$
\begin{aligned}
\frac{1}{|V|} \sqrt{\frac{k_{g}}{k_{w}}} c \inf _{u \in W^{\prime}} \int_{\Omega_{g}}\left|D u+e_{1}\right|^{2} d x \\
\quad=\frac{(1 /|V|) c k_{g} \inf _{u \in W^{\prime} \int_{\Omega_{g}}\left|D u+e_{1}\right|^{2} d x} \leq \frac{\lambda_{\text {eff }}^{+}}{\lambda_{\text {eff }}}}{\sqrt{k_{g} k_{w}}}
\end{aligned}
$$

Hence, the theorem follows if we can prove that

$$
\inf _{u \in W^{\prime}} \int_{\Omega_{g}}\left|D u+e_{1}\right|^{2} d x>0
$$

Let $Z$ denote the subspace of $L^{2}\left(\Omega_{g}, R^{2}\right)$ of the gradients of all functions in $W^{\prime}$. Since $Z$ is finite-dimensional, it is closed, and, therefore the closest approximation to $-e_{1}$ exists in $Z$, that is, we have the existence of the minimum

$$
\min _{v \in Z}\left\|v-\left(-e_{1}\right)\right\|^{2}=\min _{u \in W^{\prime}} \int_{\Omega_{g}}\left|D u+e_{1}\right|^{2} d x .
$$

Now, assume that

$$
\min _{u \in W^{\prime}} \int_{\Omega_{g}}\left|D u+e_{1}\right|^{2} d x=0
$$

(this will lead to a contradiction). Then $u=-x_{1}-1$ a.e. in $(-1,0) \times(0,1)$ and $u=-x_{1}+1$ a.e. in $(1,0) \times(0,-1)$. But then $u$ cannot belong to $W^{1,2}(V)$. To show this, let $\mathrm{F}$ be the interior of the triangle defined by the three corners $(1,0),(0,1)$ and $(0,0)$. Rotate the coordinate axis $x_{1}$ and $x_{2}$ by $45^{\circ}$ counterclockwise and denote the new coordinate axis by $\bar{x}_{1}$ and $\bar{x}_{2}$. $u$ has a representative (still denoted $u$ ) which is absolutely continuous on almost all line segments and whose partial derivatives belong to $\mathrm{L}^{2}(\mathrm{~F})$ (cf. [24, Theorem 2.1.4.]). Let $I_{t}$ be the line segment between the points $(0, t)$ and $(t, 0)$. 


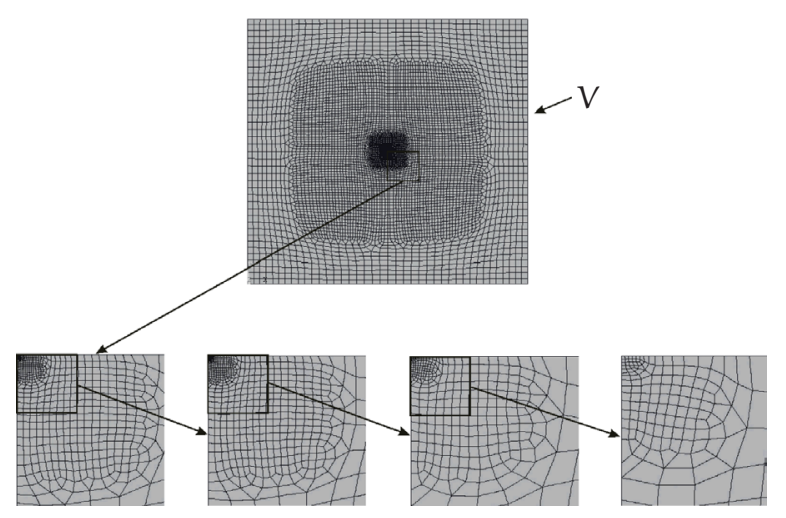

Figure 3.1. The triangulation of $\mathrm{V}$ with 28207 nodes and 9350 elements.

By the Jensen inequality

$$
\left(\frac{1}{I_{t}} \int_{I_{t}} \frac{\partial u}{\partial \bar{x}_{2}} d x_{2}\right)^{2} \leq \frac{1}{I_{t}} \int_{I_{t}}\left(\frac{\partial u}{\partial \bar{x}_{2}}\right)^{2} d x_{2}
$$

We observe that

$$
\left(\frac{1}{I_{t}} \int_{I_{t}} \frac{\partial u}{\partial \bar{x}_{2}} d x_{2}\right)^{2}=\left(\frac{2-t}{\sqrt{2} t}\right)^{2}
$$

Thus because

$$
\int_{F}\left(\frac{\partial u}{\partial \bar{x}_{2}}\right)^{2} d x=\int_{0}^{1}\left(\int_{I_{t}}\left(\frac{\partial u}{\partial \bar{x}_{2}}\right)^{2} d \bar{x}_{2}\right) \frac{d t}{\sqrt{2}},
$$

(3.9) and (3.10) give us the inequality

$$
\int_{0}^{1}\left(\frac{2-t}{\sqrt{2} t}\right)^{2} \sqrt{2} t \frac{1}{\sqrt{2}} d t \leq \int_{F}\left(\frac{\partial u}{\partial \bar{x}_{2}}\right)^{2} d x
$$

Noting that the left side is $\infty$, we obtain that $\int_{\mathrm{F}}|\mathrm{Du}|^{2} \mathrm{dx}=\infty$, which implies $\mathrm{u} \notin \mathrm{W}^{1,2}(\mathrm{~V})$, and the proof is complete.

In order to illustrate the theorem presented above we have computed the effective conductivity by a standard FEM program in the classical case, $l(r)=$ $1, k_{g}=k$ and $k_{w}=1 / k$ (concerning the finite element method in general, (cf. [3, 4]or[11]). In this case $\lambda_{\text {eff }}=1$ (see (2.5)). We have made an element 


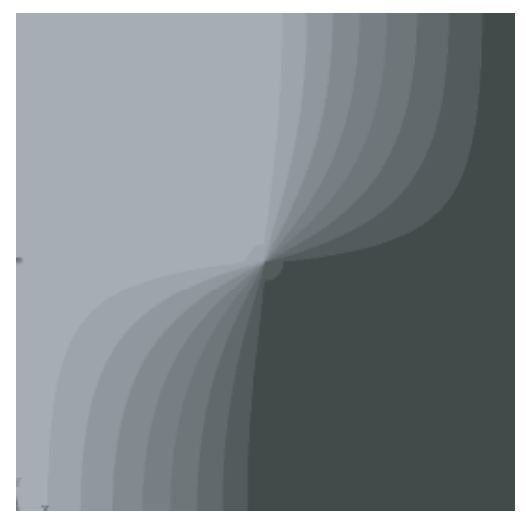

Figure 3.2. Distribution on $v$ of the function $v(x)=u(x)+x_{1}$, where $u$ is the (FEM) solution ( $v=-1$ on the left boundary and $v=1$ on the right boundary).

mesh as shown in Figure 3.1, with increasing number of elements close to the midpoint of $\mathrm{V}$. The total number of nodes and elements (quadrilateral 8-nodes elements) in this triangulation are 28207 and 9350, respectively. Even with this large number of elements we clearly see from Table 3.1 that $\lambda_{\text {eff }}^{+} / \lambda_{\text {eff }}$ diverge rapidly as $k$ increases. This agrees with the theoretical result presented in Theorem 3.1. It is important to note that not only the number of elements is critical, but also the choice of the refinement. Our choice of triangulation is based on the fact that the gradients are large near the midpoint of $\mathrm{V}$ (see the calculated temperature distribution on $\mathrm{V}$, Figure 3.2).

In Table 3.1 we present the results for some values of $k$.

Table 3.1

\begin{tabular}{llll}
\hline$k$ & $\lambda_{\text {eff }}^{-}$ & $\lambda_{\text {eff }}$ & $\lambda_{\text {eff }}^{+}$ \\
\hline 1 & 1 & 1 & 1 \\
2 & 1 & 1 & 1 \\
5 & 0.998492 & 1 & 1.00151 \\
10 & 0.947921 & 1 & 1.05497 \\
50 & 0.347867 & 1 & 2.87509 \\
100 & 0.179553 & 1 & 5.57028 \\
\hline
\end{tabular}

\section{An improved numerical method}

We will, in this section, describe a method to overcome the difficulties which arise when using the minimizer of (2.6) as an approximation of the minimizer 


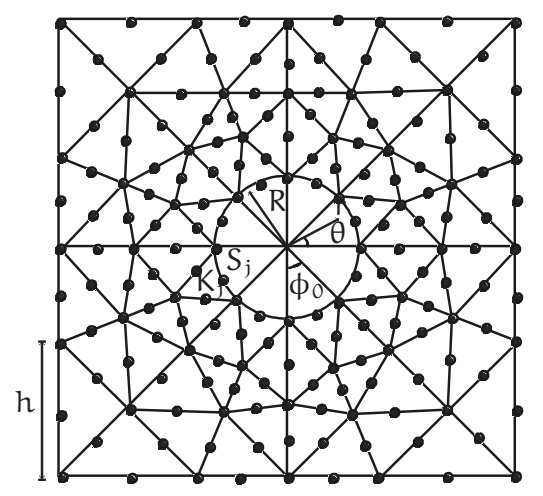

Figure 4.1. The triangulation in the new method.

of (2.2) for a finite-dimensional subspace $W^{\prime}$. This method will guarantee uniform convergence in the energy norm with respect to $k_{w} / k_{g}$ when the elementsize tends to zero.

Let $0<\alpha \leq 1$. We insert a disk $O$ in $V$ with centre in 0 and radius $R$ (see Figure 4.1). The disk $O$ is divided into equally sized sectors $\left\{S_{j}\right\}$ with angle $\phi_{0}$, on which we define the functions $\left\{t_{j}\right\}$ of the form

$$
t_{j}(r, \theta)=\frac{r^{\alpha}}{R^{\alpha}} h_{j}(\theta)
$$

where

$$
h_{j}(\theta)=a_{j} R^{2} \cos ^{2} \theta+b_{j} R^{2} \sin \theta \cos \theta+c_{j} R^{2} \sin ^{2} \theta+d_{j} R \cos \theta+e_{j} R \sin \theta+f_{j} .
$$

Moreover, on the triangular elements $\left\{K_{j}\right\}$ we define the polynomials $\left\{p_{j}\right\}$ of the form

$$
p_{j}\left(x_{1}, x_{2}\right)=a_{j} x_{1}^{2}+b_{j} x_{1} x_{2}+c_{j} x_{2}^{2}+d_{j} x_{1}+e_{j} x_{2}+f_{j} .
$$

The coefficients of $\left\{t_{j}\right\}$ and $\left\{p_{j}\right\}$ are coupled in such a way that these functions agree on common traces. For a fixed triangulation with given $R, h$ and $\phi_{0}$, where $h$ is the longest side length of the triangular elements $\left\{K_{j}\right\}$, we let $W_{h}^{\prime}$ denote the space of functions $u$ such that $u=v-x_{1} \in W$, where $v$ is antisymmetric $(v(x)=-v(-x))$, of the form $v=t_{j}$ on $S_{j}$ and $v=p_{j}$ on $K_{j}$. Now (2.6) takes the form

$$
\lambda_{\text {eff, } h}^{+}=\frac{1}{|V|} \min _{u \in W_{h}^{\prime}}\left\{\int_{V} \lambda(x)\left|\operatorname{grad} u+e_{1}\right|^{2} \mathrm{~d} x\right\} .
$$


For a given $u=v-x_{1} \in W_{h}^{\prime}$ put $h(\theta)=v(R \cos \theta, R \sin \theta)$. Using the formula

$$
|\operatorname{grad} v|^{2}=\frac{1}{r^{2}}\left(\frac{\partial v}{\partial \theta}\right)^{2}+\left(\frac{\partial v}{\partial r}\right)^{2}
$$

we obtain

$$
\begin{aligned}
\int_{V} \lambda(x)\left|\operatorname{grad} u+e_{1}\right|^{2} \mathrm{~d} x= & \int_{V} \lambda(x)|\operatorname{grad} v|^{2} \mathrm{~d} x \\
= & \frac{\mathrm{k}_{w}}{\alpha} \mathrm{K}_{1} \int_{0}^{\pi / 2}\left(\mathrm{~h}^{\prime}(\theta)\right)^{2} \mathrm{~d} \theta+\mathrm{k}_{w} \alpha \mathrm{K}_{1} \int_{0}^{\pi / 2}(\mathrm{~h}(\theta))^{2} \mathrm{~d} \theta \\
& +\frac{\mathrm{k}_{\mathrm{g}}}{\alpha} \mathrm{K}_{2} \int_{\pi / 2}^{\pi}\left(\mathrm{h}^{\prime}(\theta)\right)^{2} \mathrm{~d} \theta+\mathrm{k}_{\mathrm{g}} \alpha \mathrm{K}_{2} \int_{\pi / 2}^{\pi}(\mathrm{h}(\theta))^{2} \mathrm{~d} \theta \\
& +\int_{V \backslash \mathrm{O}} \lambda(x)|\mathrm{D} v|^{2} \mathrm{~d} x,
\end{aligned}
$$

where

$$
K_{1}=2 \alpha \int_{0}^{R}(l(r))^{-1} \frac{r^{2 \alpha-1}}{R^{2 \alpha}} d r, \quad K_{2}=2 \alpha \int_{0}^{R} l(r) \frac{r^{2 \alpha-1}}{R^{2 \alpha}} d r .
$$

In the case when $l(r)=1$ for $0 \leq r \leq R$ we obtain that $K_{1}=K_{2}=1$. We note that the integrals in (4.6) are easily calculated. For example

$$
\int_{0}^{\pi / 2}(h(\theta))^{2} d \theta=\sum_{j=0}^{M-1} \int_{j \phi_{0}}^{(j+1) \phi_{0}}\left(h_{j}(\theta)\right)^{2} d \theta
$$

where each of the integrals can be found by using standard integration formulae for trigonometric functions. Thus

$$
\int_{j \phi_{0}}^{(j+1) \phi_{0}}\left(h_{j}(\theta)\right)^{2} d \theta
$$

becomes a quadratic form in the coefficients $a_{j}, b_{j}, c_{j}, d_{j}, e_{j}$, and $f_{j}$. Summing up

$$
\int_{V} \lambda(x)|\operatorname{grad} v|^{2} \mathrm{~d} x
$$

becomes a quadratic form in the collection of all such coefficients. The minimizer of (4.4) is therefore found easily by standard numerical treatment.

In order to find an approximation of the minimizer we may solve the problem for a number of $\alpha$-values, $\alpha \in\langle 0,1]$, and search for the $\alpha$-value which gives the minimum value of

$$
\int_{V} \lambda(x)|\operatorname{grad} v|^{2} \mathrm{~d} x
$$


However, the following theorem shows that the value

$$
\alpha=\alpha_{0} \stackrel{\text { def }}{=} \frac{4}{\pi l(0)} \sqrt{\frac{k_{w}}{k_{g}}}
$$

is always a good choice.

Theorem 4.1. Let $\alpha=\alpha_{0}$. For every $\varepsilon>0$ there exist $\mathrm{R}, \phi_{0}, \mathrm{~h}>0$ so that $\left|\lambda_{\text {eff }}^{+} / \lambda_{\text {eff }}-1\right|<\varepsilon$, for every value of $k_{w} / k_{\mathrm{g}}$.

Remark 4.2. In contrast to Remark 3.2, the above theorem implies that $\left\|\mathrm{u}-\mathrm{u}^{+}\right\|_{\lambda}^{2}=\lambda_{\text {eff }}^{+}-\lambda_{\text {eff }}<\varepsilon \lambda_{\text {eff }}$ for every value of $k_{w} / k_{\mathrm{g}}$. This follows by using the Euler equations for the minimum problem (2.2).

Proof. Assume that $k_{w} / k_{\mathrm{g}} \leq 1$ (otherwise we just use the same arguments for $\left.k_{g} / k_{w}\right)$. Without loss of generality, put $k_{g}=1$, and $k_{w}=k$, hence $\lambda_{\text {eff }}=\sqrt{k}$. Let

$$
\mathrm{G}_{\mathrm{k}}(\mathrm{u})=\int_{\mathrm{V}} \lambda(x)\left|\mathrm{Du}+\mathrm{e}_{1}\right|^{2} \mathrm{~d} x
$$

First we want to prove that there exist $a>0$, and $u \in W$ such that

$$
\left|1-\frac{\mathrm{G}_{\mathrm{k}}(\mathrm{u})}{\lambda_{\mathrm{eff}, \mathrm{h}}}\right|<\varepsilon
$$

for every $0<k \leq a$. Next, we will show that there exist $R, \phi, h$, and $u \in W_{h}^{\prime}$, such that (4.14) holds for $k \in\langle 0, a]$ and such that $\left|1-\lambda_{\text {eff }}^{+} / \lambda_{\text {eff }}\right|<\varepsilon$ for every $k \in[a, 1]$. Put

$$
g(\theta)= \begin{cases}1-\frac{4}{\pi} \theta & \text { for } 0 \leq \theta \leq \frac{\pi}{2} \\ 1 & \text { for }-\frac{\pi}{2} \leq \theta<0\end{cases}
$$

and let $g(\theta)=-g(\theta-\pi)$ for $\pi / 2<\theta<3 \pi / 2$. Let $Q_{R, g}$ be the continuous function defined by $Q_{R, g}=(r / R)^{\alpha} g(\theta)$ on $O, Q_{R, g}\left(1, x_{2}\right)=1$ and $\mathrm{Q}_{\mathrm{R}, \mathrm{g}}\left(-1, x_{2}\right)=-1$. Moreover, in the grey part of $\mathrm{V} \backslash \mathrm{O}$ we let $\mathrm{Q}_{\mathrm{R}, \mathrm{g}}=1$ and -1 (on opposite quadrants), and let $Q_{R, g}$ be linear in $x_{1}$ in the white parts of $\mathrm{V} \backslash \mathrm{O}$. For the function $u=Q_{R, g}-x_{1} \in W$, we have that

$$
\mathrm{G}_{\mathrm{k}}(\mathrm{u})=\int_{V \backslash O} \lambda(x)\left|\mathrm{Du}+\mathrm{e}_{1}\right|^{2} \mathrm{~d} x+\int_{\mathrm{O}} \lambda(x)\left|\mathrm{Du}+\mathrm{e}_{1}\right|^{2} \mathrm{~d} x
$$


We observe that $|\mathrm{V}|^{-1} \int_{\mathrm{V} \backslash \mathrm{D}} \lambda(\mathrm{x})\left|\mathrm{Du}+\mathrm{e}_{1}\right|^{2} \mathrm{~d} x=\mathrm{kC}$ where $\mathrm{C}$ is independent of k. Moreover,

$$
\begin{aligned}
\int_{\mathrm{O}} \lambda(x)\left|\mathrm{Du}+\mathrm{e}_{1}\right|^{2} \mathrm{~d} x=2 \int_{0}^{\sqrt{\alpha}} & \int_{-\pi / 2}^{\pi / 2} \lambda(x)\left|\mathrm{D}\left(\mathrm{Q}_{\mathrm{R}, \mathrm{g}}\right)\right|^{2} r \mathrm{~d} r \mathrm{~d} \theta \\
& +2 \int_{\sqrt{\alpha}}^{\mathrm{R}} \int_{-\pi / 2}^{\pi / 2} \lambda(x)\left|\mathrm{D}\left(\mathrm{Q}_{\mathrm{R}, \mathrm{g}}\right)\right|^{2} \mathrm{rd} \mathrm{d} \mathrm{d} \theta
\end{aligned}
$$

We obtain

$$
\begin{aligned}
& \int_{0}^{\sqrt{\alpha}} \int_{-\pi / 2}^{\pi / 2} \lambda(x)\left|\mathrm{D}\left(\mathrm{Q}_{\mathrm{R}, \mathrm{g}}\right)\right|^{2} r \mathrm{~d} r \mathrm{~d} \theta \leq \frac{1}{\mathrm{c}_{\alpha}^{-}} \mathrm{k} \int_{0}^{\sqrt{\alpha}} \int_{0}^{\pi / 2}\left|\mathrm{D}\left(\mathrm{Q}_{\mathrm{R}, \mathrm{g}}\right)\right|^{2} \mathrm{rdr} \mathrm{d} \theta \\
& +\mathrm{c}_{\alpha}^{+} \int_{0}^{\sqrt{\alpha}} \int_{-\pi / 2}^{0}\left|\mathrm{Du}+\mathrm{e}_{1}\right|^{2} \mathrm{rdr} \mathrm{d} \theta \\
& \int_{\sqrt{\alpha}}^{R} \int_{-\pi / 2}^{\pi / 2} \lambda(x)\left|D\left(Q_{R, g}\right)\right|^{2} r d r d \theta \leq c^{+} \int_{\sqrt{\alpha}}^{1} \int_{-\pi / 2}^{0}\left|D u+e_{1}\right|^{2} r d r d \theta \\
& +\frac{1}{\mathrm{c}^{-}} k \int_{\sqrt{\alpha}}^{1} \int_{0}^{\pi / 2}\left|\mathrm{Du}+\mathrm{e}_{1}\right|^{2} \mathrm{rdr} \mathrm{d} \theta
\end{aligned}
$$

where

$$
\begin{array}{ll}
\mathrm{c}_{\alpha}^{-}=\min _{0 \leq \mathrm{r}<\sqrt{\alpha}}\{l(r)\}, & \mathrm{c}_{\alpha}^{+}=\max _{0 \leq \mathrm{r}<\sqrt{\alpha}}\{l(r)\}, \\
\mathrm{c}^{-}=\min _{0 \leq \mathrm{r}<1}\{l(r)\}, & \mathrm{c}^{+}=\max _{0 \leq \mathrm{r}<1}\{l(r)\} .
\end{array}
$$

Using the formula

$$
|\mathrm{Du}|^{2}=\left(\frac{\partial u}{\partial r}\right)^{2}+\left(\frac{\partial u}{\partial \theta}\right)^{2} \frac{1}{r^{2}}
$$

we see that the right-hand side of (4.18) and (4.19), denoted $F(g)$ and $H(g)$, can be written as

$$
\begin{aligned}
& F(g)=\frac{\alpha^{\alpha}}{R^{2 \alpha} 2 \alpha}\left(c_{\alpha}^{+} \alpha^{2} \frac{\pi}{2}+\frac{k}{c_{\alpha}^{-}} \int_{0}^{\pi / 2}\left(\alpha^{2} g(\theta)^{2}+\left(\frac{\partial g(\theta)}{\partial \theta}\right)^{2}\right) d \theta\right), \\
& H(g)=\left(\frac{1}{2 \alpha}-\frac{\alpha^{\alpha}}{R^{2 \alpha} 2 \alpha}\right)\left(c^{+} \alpha^{2} \frac{\pi}{2}+\frac{k}{c^{-}} \int_{0}^{\pi / 2}\left(\alpha^{2} g(\theta)^{2}+\left(\frac{\partial g(\theta)}{\partial \theta}\right)^{2}\right) d \theta\right),
\end{aligned}
$$


respectively. By some further calculations we obtain that

$$
\begin{aligned}
& F(g)=\sqrt{k} \frac{\alpha^{\alpha}}{R^{2 \alpha}}\left(\frac{k}{3 c_{\alpha}^{-} l(0)}+\frac{l(0)}{c_{\alpha}^{-}}+\frac{c_{\alpha}^{+}}{l(0)}\right), \\
& H(g)=\sqrt{k}\left(1-\frac{\alpha^{\alpha}}{R^{2 \alpha}}\right)\left(\frac{k}{3 c^{-} l(0)}+\frac{l(0)}{c^{-}}+\frac{c^{+}}{l(0)}\right) .
\end{aligned}
$$

Observe that $\alpha \rightarrow 0$ as $k \rightarrow 0$, which by the continuity of $l$ at 0 implies that $c_{\alpha}^{+}$and $c_{\alpha}^{-} \rightarrow l(0)$. Since $\alpha^{\alpha} \rightarrow 1$ as $k \rightarrow 0$, and

$$
\sqrt{\mathrm{k}}=\frac{1}{|\mathrm{~V}|} \min _{\mathrm{u} \in \mathrm{W}}\left\{\mathrm{G}_{\mathrm{k}}(\mathrm{u})\right\} \leq \frac{1}{|\mathrm{~V}|}(2 \mathrm{~F}(\mathrm{~g})+2 \mathrm{H}(\mathrm{g})+\mathrm{Ck})
$$

we can conclude that

$$
\frac{\mathrm{G}_{\mathrm{k}}(\mathrm{u})}{|\mathrm{V}| \sqrt{\mathrm{k}}} \longrightarrow 1 \quad \text { as } \mathrm{k} \longrightarrow 0
$$

The function $Q_{R, g}$ can be approximated by $Q_{R, g_{n}}$ in $W_{h}^{\prime}$, where

$$
g_{n}(\theta)= \begin{cases}-\frac{4}{\pi} \frac{\sin \left(\theta-\theta_{i}\right)}{\sin (\pi / 2 n)} \frac{\pi}{2 n}+1-\frac{4}{\pi} \theta_{i} & \text { for } \theta \in\left(\theta_{i}, \theta_{i+1}\right], \\ 1 & \text { for }-\frac{\pi}{2} \leq \theta<0 .\end{cases}
$$

Here, $\theta_{i}=\pi i /(2 n)$ for $i=0,1, \ldots, n-1$ and $g_{n}(\theta)=-g_{n}(\theta-\pi)$ for $\pi / 2<$ $\theta<3 \pi / 2$ (note that $\sin \left(\theta-\theta_{i}\right)=\sin \theta \cos \theta_{i}-\sin \theta_{i} \cos \theta$ ). Let $\phi=\theta_{i+1}-$ $\theta_{i}=\pi /(2 n)$, then it is easy to see that

$$
\frac{\mathrm{d}\left(\mathrm{g}_{\mathrm{n}}-\mathrm{g}\right)(\theta)}{\mathrm{d} \theta} \longrightarrow 0, \quad\left(g_{n}-\mathrm{g}\right)(\theta) \longrightarrow 0 \text { uniformly in } \theta \text { as } \phi \longrightarrow \infty \text {. }
$$

Using (4.28) in (4.22) and (4.23) we obtain from (4.25) that for every $\varepsilon>0$, there is an $\mathrm{N}$ such that

$$
\frac{\mathrm{G}_{\mathrm{k}}\left(\mathrm{u}_{\mathrm{n}}\right)}{|\mathrm{V}| \sqrt{\mathrm{k}}}-1<\varepsilon \quad \forall \mathrm{n} \geq \mathrm{N}, \forall \mathrm{k} \leq \frac{1}{\mathrm{~N}},
$$

where $u_{n}=Q_{R, g_{n}}-x_{1}$.

Let the index of $v_{k}$ denote that $v_{k}$ is a minimizer of $G_{k}$ in $W_{h}$. We want to show that for any closed subset $P \subset\langle 0,1]$, of the positive real numbers, there exist $R, \phi$, and $h$ such that,

$$
\left|1-\frac{\mathrm{G}_{\mathrm{k}}\left(v_{\mathrm{k}}\right)}{\sqrt{\mathrm{k}}}\right|<\varepsilon \quad \text { holds } \forall \mathrm{k} \in \mathrm{P} .
$$

Put $k^{-}=\min _{k \in P}\{k\}$ and put $A^{+}=\max _{k \in P}\left\{G_{k}\left(v_{k}\right)\right\}<\infty$. Choose a finite subset $J$ of $P$ such that for every $k \in P$ there is some $y \in J$, such that $k \geq y$ 
and $k-y=\Delta t<\delta /\left(x^{-} A^{+}\right)$. Choose $R, \phi$ and $h$ so small (using standard theory for error estimates in the finite element method, compare, e.g., with [11, page 97]) that

$$
\left|1-\frac{\mathrm{G}_{\mathrm{y}}\left(\nu_{\mathrm{y}}\right)}{\sqrt{\mathrm{y}}}\right|<\frac{\varepsilon}{2} \quad \forall \mathrm{y} \in \mathrm{J} .
$$

Let $k$ be some arbitrary point in $P$ and let $y \in J$ be such that,

$$
k \geq y, \quad k-y=\Delta t<\frac{\delta}{x^{-} A^{+}} .
$$

Put

$$
\begin{aligned}
& A(v)=\frac{2}{|V|} \int_{0}^{1} \int_{0}^{1} \lambda\left(x_{1}, x_{2}\right)\left|\mathrm{D} v+e_{1}\right|^{2} d x \\
& B(v)=\frac{2}{|V|} \int_{0}^{-1} \int_{0}^{1} \lambda\left(x_{1}, x_{2}\right)\left|D v+e_{1}\right|^{2} d x .
\end{aligned}
$$

It follows that

$$
\begin{aligned}
\mathrm{G}_{y}\left(v_{y}\right) & \leq \mathrm{G}_{k}\left(v_{k}\right) \leq \mathrm{G}_{k}\left(v_{y}\right)=\mathrm{A}\left(v_{y}\right)+\mathrm{B}\left(v_{y}\right) \\
& =\frac{(\Delta t+y)}{k} \mathrm{~A}\left(v_{y}\right)+\mathrm{B}\left(v_{y}\right) \leq \mathrm{G}_{y}\left(v_{y}\right)+\frac{\Delta t}{k} \mathrm{~A}\left(v_{y}\right)<\mathrm{G}_{y}\left(v_{y}\right)+\delta .
\end{aligned}
$$

Hence, $G\left(k, v_{k}\right)-G\left(y, v_{y}\right)<\delta$. Since the function $k \mapsto 1 / \sqrt{k}$ is uniformly continuous in $P$, there is a $\delta>0$ such that

$$
\left|\frac{G_{y}\left(v_{y}\right)}{\sqrt{y}}-\frac{G_{k}\left(v_{k}\right)}{\sqrt{k}}\right|<\frac{\varepsilon}{2}
$$

holds for every $k \in P$, hence from (4.31) and (4.36), we conclude that (4.30) holds.

Put $Q_{R, g_{n}}-x_{1}=u_{R, n}$. Observe from (4.29) that we can obtain $k_{1} \in(0,1]$ and $n_{1}$ such that $G_{x}\left(u_{1, n_{1}}\right) / \sqrt{k}-1<\varepsilon / 2$ holds for every $k<k_{1}$. By (4.30) we can find $R=R_{1}, h=h_{1}$, and $\phi=\phi_{0,1}$ such that $G_{k}\left(v_{k}\right) / \sqrt{k}-1<\varepsilon$ for every $k \in\left[k_{1}, 1\right]$. Again by (4.29), there is a number $k_{2}, 0<k_{2}<k_{1}$, and $n_{2} \geq n_{1}$ such that

$$
\frac{\mathrm{G}_{\mathrm{k}}\left(\mathrm{u}_{\mathrm{R}_{1}, \mathrm{n}_{2}}\right)}{\sqrt{\mathrm{k}}}-1<\frac{\varepsilon}{2}
$$

holds for every $k<k_{2}$. Hence, there is $h=h_{2}$ and $\phi=\phi_{0,2}$ such that $\mathrm{G}_{\mathrm{k}}\left(v_{\mathrm{k}}\right) / \sqrt{\mathrm{k}}-1<\varepsilon$ holds for every $\mathrm{k}<\mathrm{k}_{2}$. Let $\mathcal{F} \subset \mathrm{W}_{\mathrm{h}}$ consists (for $\mathrm{h}=\mathrm{h}_{2}$, $\left.R=R_{1}, \phi_{0}=\phi_{0,2}\right)$ of the functions in $W_{h}$ that is equal to $u_{1, n_{2}}$ inside the 


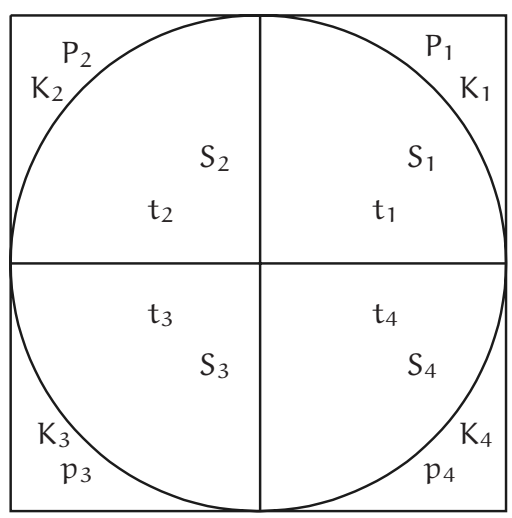

Figure 4.2. The simplest case of triangulation with the new method.

disc of radius $R$, and let $q_{k}$ denote a minimizer of $G_{k}$ in $\mathcal{F}$. Observe that for any $k \leq k_{1}$ it holds that

$$
\frac{\mathrm{G}_{\mathrm{k}}\left(\mathrm{u}_{1, \mathrm{n}_{2}}\right)}{\sqrt{\mathrm{k}}}-1<\frac{\varepsilon}{2}
$$

Hence, by (4.30) there is $h=h_{2}$ and $\phi=\phi_{0,3}$ such that $\left|1-G_{k}\left(q_{k}\right) / \sqrt{k}\right|<\varepsilon$ holds for every $k_{2} \leq k \leq k_{1}$, thus $\left|1-G_{k}\left(v_{k}\right) / \sqrt{k}\right|<\varepsilon$. Put

$$
R=R_{1}, \quad \phi=\min \left\{\phi_{0,1}, \phi_{0,2}, \phi_{0,3}\right\}, \quad h=\min \left\{h_{1}, h_{2}\right\} .
$$

Then, $G_{k}\left(v_{k}\right) / \sqrt{k}-1<\varepsilon$ holds for every $k>0$. This completes the proof.

\section{An illustrative example}

For the sake of illustration put $l(r)=1, k_{g}=k, k_{w}=1 / k$, and consider the simple triangulation shown in Figure 4.2.

Without presenting the detailed calculation we compute an upper estimate for $\lambda_{\text {eff, }}^{+}$by choosing the function $u=v-x_{1} \in W_{h}^{\prime}$ where $v$ is antisymmetric $(v(x)=-v(-x))$, of the form $v=t_{j}$ on $S_{j}$ and $v=p_{j}$ on $K_{j}$. Here,

$$
\begin{gathered}
\mathrm{t}_{1}=-\mathrm{t}_{3}=\frac{\mathrm{r}^{\alpha} \cos 2 \theta}{\mathrm{R}^{\alpha}}\left(=\frac{\mathrm{r}^{\alpha}\left(2 \cos ^{2} \theta-1\right)}{\mathrm{R}^{\alpha}}\right), \quad \mathrm{t}_{2}=-\mathrm{t}_{4}=-\frac{\mathrm{r}^{\alpha}}{\mathrm{R}^{\alpha}}, \\
\mathrm{p}_{1}=-\mathrm{p}_{3}=2 x_{1}^{2}-1, \quad \mathrm{p}_{2}=-\mathrm{p}_{4}=-1 .
\end{gathered}
$$


We obtain

$$
\begin{aligned}
& \frac{1}{|\mathrm{~V}|} \int_{\mathrm{V}} \lambda(x)|\operatorname{grad} v|^{2} \mathrm{~d} x \\
& \quad=\frac{2}{|\mathrm{~V}|} \int_{\mathrm{S}_{1} \cup \mathrm{S}_{4}} \lambda(x)|\operatorname{grad} v|^{2} \mathrm{~d} x+\frac{2}{|\mathrm{~V}|} \int_{\mathrm{K}_{1} \cup \mathrm{K}_{4}} \lambda(x)|\operatorname{grad} \nu|^{2} \mathrm{~d} x .
\end{aligned}
$$

It is easily seen that

$$
\int_{K_{1} \cup K_{4}} \lambda(x)|\operatorname{grad} v|^{2} \mathrm{~d} x=\frac{1}{\mathrm{k}}\left(\frac{16}{3}-\pi\right) .
$$

Moreover,

$$
\begin{aligned}
\int_{S_{1} \cup S_{4}} \lambda(x)|\operatorname{grad} v|^{2} \mathrm{~d} x & =\int_{-\pi / 2}^{\pi / 2} \int_{0}^{1} \lambda(x)\left(\frac{1}{r^{2}}\left(\frac{\partial v}{\partial \theta}\right)^{2}+\left(\frac{\partial v}{\partial r}\right)^{2}\right) r d r d \theta \\
& =\frac{1}{k}\left(\frac{\pi}{\alpha}+\frac{\pi}{8 \alpha}\left(\alpha^{2}-4\right)\right)+k\left(\frac{\pi}{4} \alpha\right) .
\end{aligned}
$$

Thus

$$
\frac{1}{|\mathrm{~V}|} \int_{\mathrm{V}} \lambda(x)|\operatorname{grad} \nu|^{2} \mathrm{~d} x=\frac{1}{2 \mathrm{k}}\left(\frac{16}{3}-\pi+\frac{1}{8} \pi \alpha+\frac{\pi}{2 \alpha}\right)+\mathrm{k} \frac{\pi}{8} \alpha
$$

Setting

$$
\alpha=\frac{4}{\pi l(0)} \sqrt{\frac{k_{w}}{k_{g}}}=\frac{4}{\pi k},
$$

we obtain from (5.5) that

$$
\lambda_{\text {eff, } h}^{+} \leq \frac{1}{|V|} \int_{V} \lambda(x)|\operatorname{grad} v|^{2} \mathrm{~d} x=\frac{1}{4 \mathrm{k}^{2}}+\frac{8}{3 \mathrm{k}}-\frac{\pi}{2 \mathrm{k}}+\frac{\pi^{2}}{16}+\frac{1}{2} .
$$

Remark 5.1. We recall that the exact value $\lambda_{\text {eff }}=1$. In our example, we only use 4 elements outside the disk O. From (5.7) we obtain that $\lambda_{\text {eff, } h}^{+} \leq 1.12783$ when $k=100$. Note that the corresponding value from the standard numerical treatment (with 9350 elements) in Section 3 is 5.57028 (see Table 3.1). This illustrates the power of our new method.

Remark 5.2. In the special case of standard checkerboards we have only compared our method with calculations obtained from using the finite element method (FEM). However, there exist methods based on solving integral equations numerically which are significantly better than standard FEM in case of difficult geometries. Here we want to refer to a recent work of Helsing [8] which considers checkerboards in which the white squares (with conductivity $=100$ ) are a little bit smaller than the darker ones (with 
conductivity $=1$ ). In particular, by his method he has calculated the effective conductivity to be 9.89299 in the case when the white squares occupy $49,9999999 \%$ of the whole structure. According to Helsing it is also possible to apply a modified version of his method in case of standard checkerboards.

\section{Some final comments}

We think that the results obtained in this paper will be useful for mathematicians and engineers dealing with checkerboard composites. Our numerical experiments show that it is difficult to obtain good approximations by using the finite element method, and Theorem 3.1 (see also Remark 3.2) even states that there exists no finite-dimensional function space which can approximate the actual solution (independently of the material properties in the checkerboard).

By the new method we overcome this obstacle (see Theorem 4.1). It seems to be possible to extend the method to 4-phase checkerboards. We aim to develop these ideas in a forthcoming paper.

\section{Acknowledgement}

We thank J. Helsing and G. W. Milton for stimulating discussions and generous advises which have improved the final version of this paper.

\section{References}

[1] V. L. Berdichevskii, Heat conduction in checkerboard grids, Vestnik Moskov. Univ. Ser. I Mat. Mekh. (1985), no. 4, 56-63 (Russian). MR 87b:80003.

[2] L. Berlyand and K. Golden, Exact result for the effective conductivity of a continuum percolation model, Physical Review B: Solid State 50 (1994), 21142117.

[3] P. G. Ciarlet and J. L. Lions (eds.), Handbook of Numerical Analysis. Vol. II. Finite Element Methods. Part 1, North-Holland, Amsterdam, 1991. MR 92f:65001. Zbl 0712.65091.

[4] P. G. Ciarlet and J. L. Lions (eds.), Handbook of Numerical Analysis. Vol. IV. Finite Element Methods. Part 2, Numerical Methods for Solids. Part 2, North-Holland, Amsterdam, 1995. Zbl 0864.65001.

[5] R. V. Craster and Y. V. Obnosov, Four-phase checkerboard composites, SIAM J. Appl. Math. 61 (2001), no. 6, 1839-1856. CMP 1856873.

[6] A. M. Dychne, Conductivity of a two-phase two-dimensional system, Exper. and Theor. Phys. 59 (1970), no. 7, 110-115.

[7] K. M. Golden and S. M. Kozlov, Critical path analysis of transport in highly disordered random media, Homogenization (v. Berdichevsky and G. Papanicolaou, eds.), Advances in Mathematics for Applied Sciences, vol. 50, World Scientific Publishing, New Jersey, 1999, pp. 21-34. MR 2001k:82097. 
[8] J. Helsing, Corner singularities for elliptic problems: special basis functions versus brute force, Commun. Numer. Methods Eng. 16 (2000), 37-46. Zbl 0947.65126.

[9] V. V. Jikov, S. M. Kozlov, and 0. A. Oleuinik, Homogenization of Differential Operators and Integral Functionals, Springer-Verlag, Berlin, 1994. MR 96h:35003b.

[10] V. V. Jikov and D. Lukkassen, On two types of effective conductivities, J. Math. Anal. Appl. 256 (2001), no. 1, 339-343. CMP 1820 085. Zbl 01620003.

[11] C. Johnson, Numerical Solution of Partial Differential Equations by the Finite Element Method, Studentlitteratur, Lund, 1987. MR 89b:65003b. Zbl 0628.65098.

[12] J. B. Keller, Effective conductivity of periodic composites composed of two very unequal conductors, J. Math. Phys. 28 (1987), no. 10, 2516-2520. Zbl 0634.73054.

[13] S. M. Kozlov, Geometric aspects of averaging, Russ. Math. Surv. 44 (1989), no. 2, 91-144, translated from Uspekhi Mat. Nauk 44 (1989), no.2(266), 79 120. MR 91a:58163. Zbl 0706.49029.

[14] D. Lukkassen, Homogenization of integral functionals with extreme local properties, Math. Balkanica (N.S.) 12 (1998), no. 3-4, 339-358. MR 2000f:35016.

[15] G. W. Milton, Theoretical studies of the transport properties of inhomogeneous media, unpublished report TP/79/1, 1979.

[16] , Proof of a conjecture on the conductivity of checkerboards, J. Math. Phys. 42 (2001), no. 10, 4873-4882. CMP 1855103.

[17] V. V. Mityushev and T. N. Zhorovina, Exact solution of the problem of current formation in a doubly periodic heterogeneous system, Boundary Value Problems, Special Functions and Fractional calculus (Russian) (Minsk, 1996), Belorus. Gos. Univ., Minsk, 1996, pp. 237-243. CMP 1428946.

[18] S. Mortola and S. Steffe, A two-dimensional homogenization problem, Atti Accad. Naz. Lincei Rend. Cl. Sci. Fis. Mat. Natur. (8) 78 (1985), no. 3, 77-82. MR 89f:49024.

[19] Y. V. Obnosov, Exact solution of a boundary-value problem for a rectangular checkerboard field, Proc. Roy. Soc. London Ser. A 452 (1996), no. 1954, 2423-2442. MR 97j:73002. Zbl 0891.73047.

[20] _ Periodic heterogeneous structures: new explicit solutions and effective characteristics of refraction of an imposed field, SIAM J. Appl. Math. 59 (1999), no. 4, 1267-1287. Zbl 0936.30029.

[21] P. Sheng and R. V. Kohn, Geometric effects in continuous-media percolation, Physical Review B (Solid State) 26 (1982), 131-1335.

[22] L. Tartar, An introduction to the homogenization method in optimal design, Optimal Shape Design (Tróia, 1998), Lecture Notes in Math., vol. 1740, Springer, Berlin, 2000, pp. 47-156. CMP 1804 685. Zbl 01574749.

[23] V. V. Zhikov, Passage to the limit in nonlinear variational problems, Russ. Acad. Sci., Sb., Math. 76 (1993), no. 2, 427-459, translated from Mat. Sb. 183 (1992), no. 8, 47-84. MR 93i:49016. Zbl 0791.35036.

[24] W. P. Ziemer, Weakly Differentiable Functions. Sobolev Spaces and Functions of Bounded Variation, Graduate Texts in Mathematics, vol. 120, SpringerVerlag, New York, 1989. MR 91e:46046. Zbl 0692.46022. 
Stein A. Berggren et al. 173

Stein A. Berggren: Narvik University College, P.0. Box 385, N-8505 Narvik, Norway E-mail address: sab@hin.no

Dag Lukkassen: Narvik University College, P.0. Box 385, N-8505 Narvik, Norway E-mail address: dag.lukkassen@hin.no

Annette Meidell: Narvik University College, P.0. Box 385, N-8505 Narvik, Norway E-mail address: am@hin.no

Leon Simula: Narvik University College, P.0. Box 385, N-8505 Narvik, Norway E-mail address: leons@hin.no 


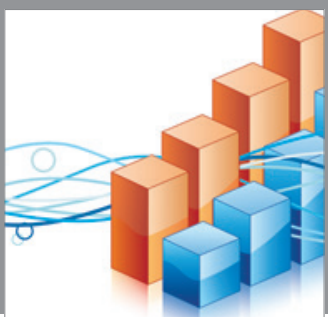

Advances in

Operations Research

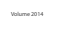

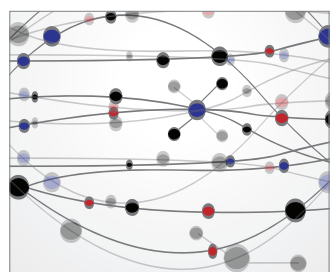

\section{The Scientific} World Journal
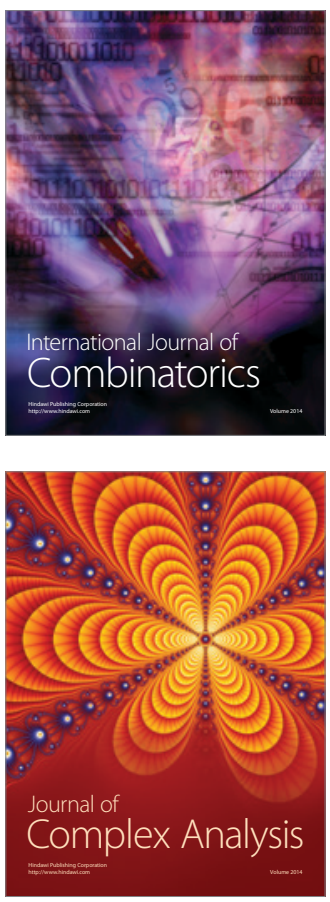

International Journal of

Mathematics and

Mathematical

Sciences
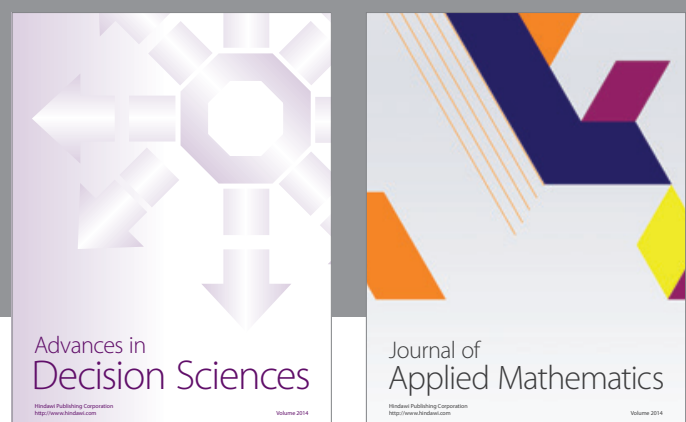

Journal of

Applied Mathematics
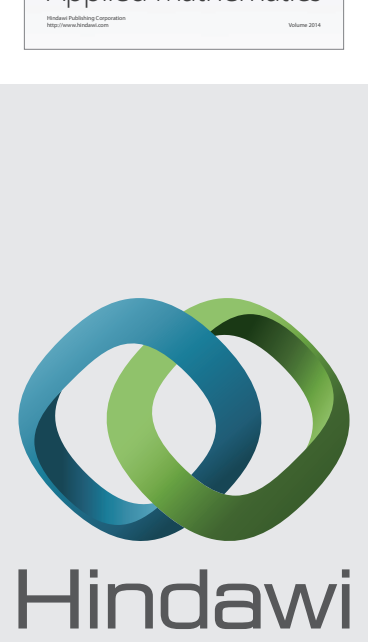

Submit your manuscripts at http://www.hindawi.com
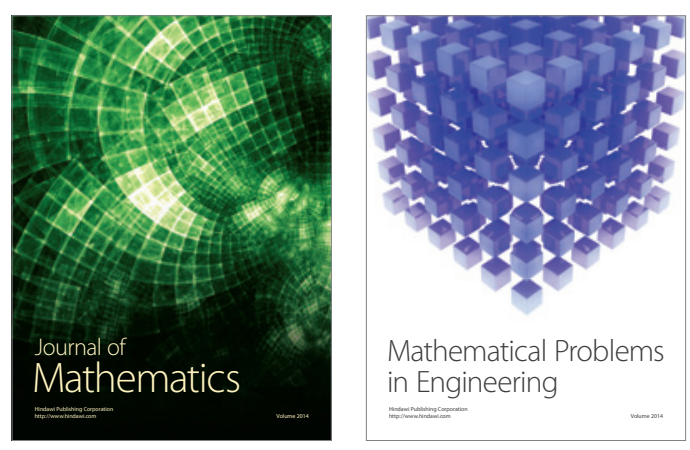

Mathematical Problems in Engineering
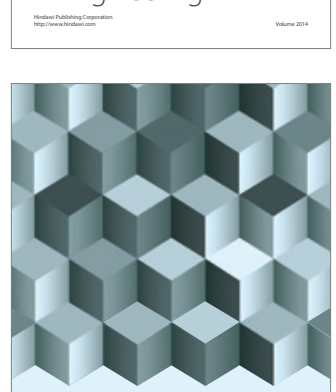

Journal of

Function Spaces
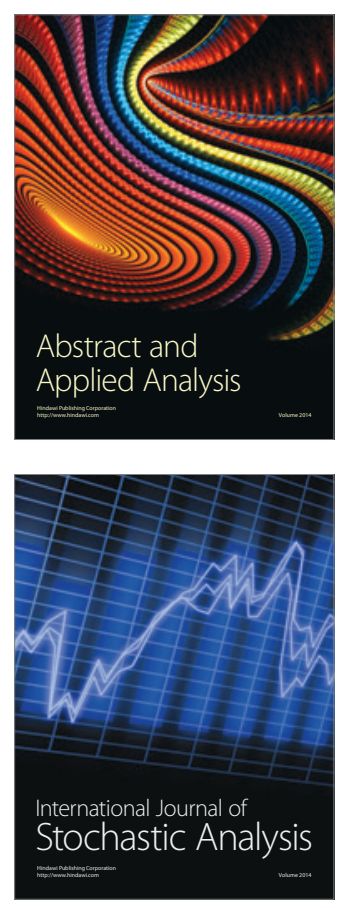

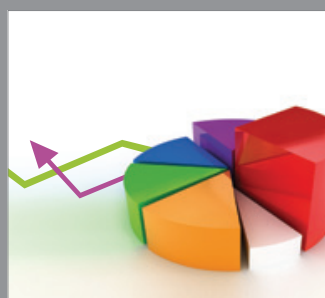

ournal of

Probability and Statistics

Promensencen
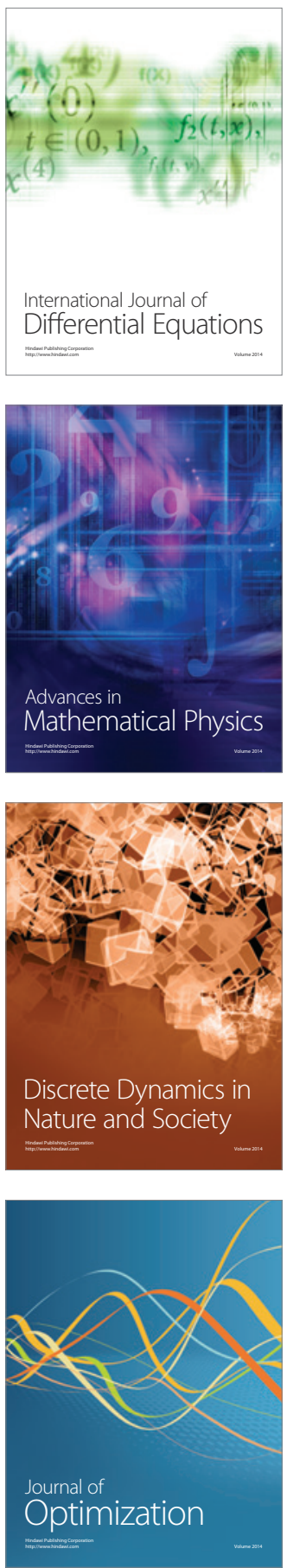